\title{
Device Identifiers Domain
}

National Cancer Institute

\section{Source}

National Cancer Institute. Device Identifiers Domain. NCI Thesaurus. Code C102618.

A subject domain utilized for the submission of information encompassing and representing data, vocabulary or records related to device identifiers. 